\title{
Perspective: Vitamin D supplementation prevents rickets and acute respiratory infections when given as daily maintenance but not as intermittent bolus: implications for COVID-19
}

\author{
Authors: George Griffin, ${ }^{\mathrm{A}}$ Martin Hewison, ${ }^{\mathrm{B}}$ Julian Hopkin, ${ }^{\mathrm{C}}$ Rose Anne Kenny, ${ }^{\mathrm{D}}$ Richard Quinton, ${ }^{\mathrm{E}}$ Jonathan Rhodes, ${ }^{\mathrm{F}}$ \\ Sreedhar Subramanian ${ }^{G}$ and David Thickett ${ }^{H}$
}

The value of vitamin $D$ supplementation in the treatment or prevention of various conditions is often viewed with scepticism as a result of contradictory results of randomised trials. It is now becoming apparent that there is a pattern to these inconsistencies. A recent large trial has shown that high-dose intermittent bolus vitamin $D$ therapy is ineffective at preventing rickets - the condition that is most unequivocally caused by vitamin $D$ deficiency. There is a plausible biological explanation since high-dose bolus replacement induces long-term expression of the catabolic enzyme 24-hydroxylase and fibroblast growth factor 23, both of which have vitamin D inactivating effects. Meta-analyses of vitamin $D$ supplementation in prevention of acute respiratory infection and trials in tuberculosis and other conditions also support efficacy of low dose daily maintenance rather than intermittent bolus dosing. This is particularly relevant during the current COVID-19 pandemic given the well-documented associations between COVID-19 risk and vitamin D deficiency. We would urge that clinicians take note of these findings and give strong support to widespread use of daily vitamin D supplementation.

KEYWORDS: vitamin D, COVID-19, maintenance, dosing, efficacy

DOI: $10.7861 /$ clinmed.2021-0035

Authors: Aemeritus professor of infectious diseases and medicine, St George's, University of London, London, UK; Bprofessor of molecular endocrinology, University of Birmingham, Birmingham, UK; Crector of medicine and health and professor of experimental medicine, Swansea University, Swansea, UK; D professor of medical gerontology, Trinity College Dublin, Dublin, Ireland; 'Esenior lecturer in endocrinology, Newcastle University, Newcastle upon Tyne, UK; Femeritus professor of medicine, University of Liverpool, Liverpool, UK; ${ }^{6}$ consultant gastroenterologist, Royal Liverpool University Hospital, Liverpool, UK; "Hprofessor in respiratory medicine, University of Birmingham, Birmingham, UK
Introduction: contradictory results of vitamin D supplementation in clinical trials

Controversy surrounding the possible benefits from taking vitamin $\mathrm{D}$ supplements has become more prominent recently with the reports of various associations between vitamin $D$ deficiency and risk of COVID-19. Authorities have given only muted support to avoidance of vitamin D deficiency during the current COVID-19 pandemic, concluding that there is insufficient evidence from randomised trials of supplementation to support any protective effect against COVID-19. ${ }^{2}$ Many randomised trials of vitamin $D$ supplementation have been performed in a wide range of conditions with contradictory and often negative results that have led to considerable scepticism about its value. ${ }^{3,4}$ This may partly result from optimistic trial of vitamin D supplementation in widely diverse conditions, not always with a plausible mechanism of causation. It is becoming apparent, though, that the use of intermittent high-dose bolus, introduced to achieve high adherence, rather than regular daily maintenance could be an important explanation for the failure of vitamin $D$ supplementation in some trials.

Evidence that low-dose daily vitamin D supplementation is effective but intermittent high-dose bolus is not

Rickets

Rickets is the one condition above all others where there is conclusive and very longstanding evidence, dating back to the 1920s, first in animals and then in children, of response to regular daily vitamin $D$, either via cod liver oil supplementation or later by food fortification. ${ }^{5}$ A recent well-conducted trial in 3,046 children has however shown that high-dose bolus vitamin D (100,000 IU every 3 months for 18 months) is ineffective at preventing rickets in children ${ }^{6}-$ indeed it is not the first trial to show this. ${ }^{7}$ A smaller study in 72 children with calcium-deficiency rickets showed that oral vitamin D2 50,000 IU monthly for 24 weeks had only a 'marginally significant effect' $(p=0.06)$ on recovery compared with calcium supplementation alone. ${ }^{8}$ 


\section{Tuberculosis}

Tuberculosis is another condition for which there is longstanding evidence suggesting that vitamin $D$ deficiency contributes to illness and susceptibility. As with rickets, this dates back a long way, a successful trial of daily cod liver oil in tuberculosis having been reported in 1848 from London's Brompton Hospital. ${ }^{9}$ However, although ultraviolet light and vitamin D therapy were well-established therapies, particularly in cutaneous tuberculosis, during the first half of the 20th century, controlled trials were not performed.$^{10} \mathrm{~A}$ recent meta-analysis of vitamin $\mathrm{D}$ supplementation as an adjunct to chemotherapy for active pulmonary tuberculosis showed significantly accelerated sputum conversion but only in patients with multi-drug-resistant tuberculosis. In this analysis, only one of seven included studies trialled daily vitamin D supplementation. ${ }^{11}$ No significant difference was seen for daily or weekly supplementation combined versus bolus/2-weekly regimens. However, the single trial of daily supplementation (5,000 IU/day) versus placebo in 288 patients with active pulmonary tuberculosis did show significant benefit across the whole patient population, with $61.3 \%$ of patients culture-negative at 4 weeks compared with $42.2 \%$ placebo $(p=0.032) .^{12}$

In a 2015 review of vitamin D supplementation as prophylaxis against tuberculosis in high-risk individuals, it was noted that no randomised trials had yet taken place despite consistent evidence that vitamin $\mathrm{D}$ deficiency increases risk for developing active tuberculosis. ${ }^{13}$ There had however been one study looking at the effect of supplementation with 800 IU/day over 6 months in 120 children from Mongolia, all with vitamin D deficiency $(<50 \mathrm{nmol} / \mathrm{L})$, which showed a trend towards fewer tuberculin skin test conversions (risk ratio [RR] 0.41, 95\% confidence interval [CI] 0.16-1.09; $p=0.06)$ and a significantly greater increase in stature $(p<0.003) .{ }^{14}$ Recently, a much larger trial in 8,851 children from Mongolia, 95.6\% with vitamin D deficiency ( $<50 \mathrm{nmol} / \mathrm{L}$ ), but this time using a weekly oral dose of 14,000 IU vitamin D3 compared with placebo, showed no impact on subsequent diagnosis of tuberculosis (adjusted RR $0.87,95 \%$ CI $0.49-1.55$ ) or on risk of hospitalisation with acute respiratory infection (adjusted RR $0.86,95 \%$ CI $0.52-1.40$ )..$^{15}$

\section{Acute respiratory infections}

The recent meta-analyses by Martineau and colleagues of the efficacy of vitamin D supplementation in prevention of acute respiratory infections have also reported that low dose daily maintenance supplementation is effective but intermittent high-dose bolus is not. 16,17 In their most recent meta-analysis of 42 randomised controlled trials, 47,262 subjects were analysed. A protective effect against risk for acute respiratory infection was seen for trials in which vitamin D supplements were given daily (odds ratio [OR] $0.70,95 \%$ CI $0.61-0.93$ ) but not for trials where vitamin D was given weekly (OR 0.97, 95\% CI 0.88-1.06) or monthly to 3-monthly (OR 0.98, 95\% CI 0.93-1.03). ${ }^{17}$

Thus for treatment or prevention of rickets, tuberculosis and acute respiratory infections, there is substantial evidence that it is daily vitamin $D$ supplementation that is effective rather than intermittent high-dose boluses.

\section{A plausible biological mechanism underlies the lack of efficacy of intermittent high-dose (bolus) vitamin D}

Vitamin $D$ (cholecalciferol) is synthesised in the skin by the action of ultraviolet B (UVB, wavelength 280-315 nm) on exposed skin, splitting a carbon-to-carbon bond (C9-C10) in the precursor 7-dehydrocholesterol. ${ }^{18}$ Cholecalciferol is 25 -hydroxylated in the liver and subsequently $1 \alpha$-hydroxylated in the kidneys and in other tissues including immune cells and many epithelia to form the active metabolite 1,25 dihydroxycholecalciferol (1,25(OH)2D). The predominant circulating vitamin $D$ is 25 -hydroxycholecalciferol $(25(\mathrm{OH}) \mathrm{D})$, which is itself a substrate for local activation in tissues expressing the $1 \alpha$-hydroxylase enzyme and is present in nanomolar concentrations, whereas circulating active $1,25(\mathrm{OH}) 2 \mathrm{D}$ is only present in picomolar concentrations. Like other lipophilic molecules, $25(\mathrm{OH}) \mathrm{D}$ is bound to protein in serum, approximately $80 \%$ to the vitamin D binding protein (DBP) and $20 \%$ to albumin. ${ }^{19}$ Given that the vitamin $\mathrm{D}$ receptor (VDR) is predominantly a nuclear receptor, it is the intracellular concentration of $1,25(\mathrm{OH}) 2 \mathrm{D}$ that is most relevant and most/all cells that express the VDR, including osteoblasts and immune cells, also express $1 \alpha$-hydroxylase activity, so circulating concentrations of $1,25(\mathrm{OH}) 2 \mathrm{D}$ may not always reflect intracellular concentrations. Currently, almost all studies of vitamin $D$ rely on the measurement of serum $25(\mathrm{OH}) \mathrm{D}$ to determine vitamin $\mathrm{D}$ status but, given the complexities of variable protein binding in illness plus its complex metabolism, there is a need to consider other metabolites such as 1,25(OH)2D and 24,25(OH)2D, particularly in the setting of different dosing regimens. The half-life of $25(\mathrm{OH}) \mathrm{D}$ in the blood is around $2-3$ weeks but the whole-body half-life is around 2-3 months, probably because cholecalciferol, which is lipid-soluble, is conserved in body fat. ${ }^{20}$ As a result it has been widely presumed that large intermittent boluses, which provide relatively stable $25(\mathrm{OH}) \mathrm{D}$ serum concentrations, could provide effective replacement. ${ }^{21}$

The metabolism of vitamin D is complex, however, and still incompletely understood (Fig 1). A large amount of cholecalciferol

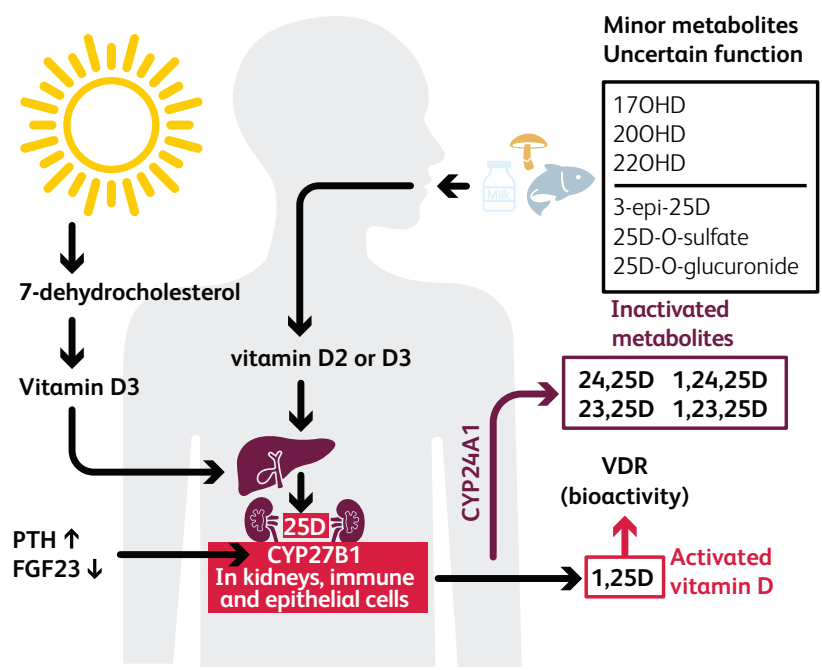

Fig 1. The complexity of vitamin D metabolism. D2 is from plant sources via ultraviolet action on ergosterol and D3 from animal sources via ultraviolet action on 7-dehydrocholesterol. Activation is via 25 -hydroxylation in the liver followed by $1 \alpha$-hydroxylation (CYP27B1) in kidneys, immune cells and many epithelia, to 1,25(OH)2D. Increased FGF23 suppresses $1 \alpha$-hydroxylation. Free $25(\mathrm{OH}) \mathrm{D}$ appears to be preferentially taken up by monocytes, 19 so the reduction in DBP in illness may have a protective effect via increased availability of free $25(\mathrm{OH}) \mathrm{D}$. Either $25(\mathrm{OH}) \mathrm{D}$ or 1,25(OH)2D can be degraded via 24-hydroxylation (CYP24A1) to $24,25(\mathrm{OH}) \mathrm{D}$ or $1,24,25(\mathrm{OH}) 3 \mathrm{D}$ respectively. 
can be generated rapidly by sunlight exposure - one full-body UV exposure causing slight skin pinkness is equivalent to $250-625 \mu \mathrm{g}$ (10,000-25,000 IU) vitamin D $3,{ }^{18}$ whereas total daily requirement is only around $800-1,000 \mathrm{IU}^{22}$ - so the level of activated vitamin $\mathrm{D}$ in the body could potentially vary enormously without robust regulatory mechanisms.

It is impossible for vitamin D toxicity to occur through ultraviolet exposure (and extremely high repeated dosing is required for this to occur via supplementation). The principal regulatory check is via 24-hydroxylation forming $24,25(\mathrm{OH}) 2 \mathrm{D}$ or $1,24,25(\mathrm{OH}) 3 \mathrm{D}$, which are both largely inactive. The inactivating enzyme 24 -hydroxylase CYP24A1 catalyses not only 24-hydroxylation but also 23-hydroxylation, generating a series of polyhydroxylated products. Although these may have some biological activity, the importance of this process in inactivating vitamin $D$ is demonstrated by the finding that inborn mutations of CYP24A1 cause idiopathic infantile hypercalcemia. ${ }^{23}$ CYP24A1 is expressed in cells containing the vitamin $\mathrm{D}$ receptor, including kidney, bone, intestine and dendritic cells, and increases markedly in response to rising levels of $25(\mathrm{OH}) \mathrm{D} .{ }^{24}$ The intracellular balance between the activating $1 \alpha$ hydroxylase CYP27B1 and the inactivating 24-hydroxylase CYP24A1 is thought to be critically important, particularly in extraskeletal tissues, for regulating activity of vitamin D. ${ }^{25}$
It has only been recognised relatively recently that the increased 24-hydroxylase activity, as a feedback control response to a large bolus of vitamin D, may itself have a long half-life - persisting for several weeks after the bolus dose ${ }^{26}$ (Fig 2). This implies that a single high-dose bolus of vitamin D could paradoxically lead to intracellular deficiency of activated vitamin $D$ as a rebound phenomenon. This might be particularly important in immune cells such as the dendritic cells that are probably central to the hyperinflammatory state seen in severe COVID-19 and that seem particularly sensitive to this process. ${ }^{24}$

The role of fibroblast growth factor 23 (FGF23) in vitamin D regulation may be at least equally important in regulating activation of vitamin $\mathrm{D}$. Its expression is increased in the presence of high serum 25(OH)D and 1,25(OH)D concentrations. A meta-analysis of 23 studies has shown that vitamin $\mathrm{D}$ supplementation daily or at an equivalent of $\leq 2,000 \mathrm{IU} /$ day had no significant effect on circulating FGF23 concentrations, whereas higher doses resulted in a significant increase (FGF23 +18 pg/ml, 95\% CI 6-30), particularly when circulating $25(\mathrm{OH}) \mathrm{D}$ achieved was $\geq 100 \mathrm{nmol} / \mathrm{L}(\mathrm{p}<0.001) .{ }^{27}$ The likely importance of this increase in FGF23 in the suppression of vitamin $D$ activation is demonstrated by the condition tumour-associated osteomalacia, in which inappropriate FGF23 secretion by tumour tissue resulting in serum concentrations of FGF23 $>30 \mathrm{pg} / \mathrm{ml}$ in the
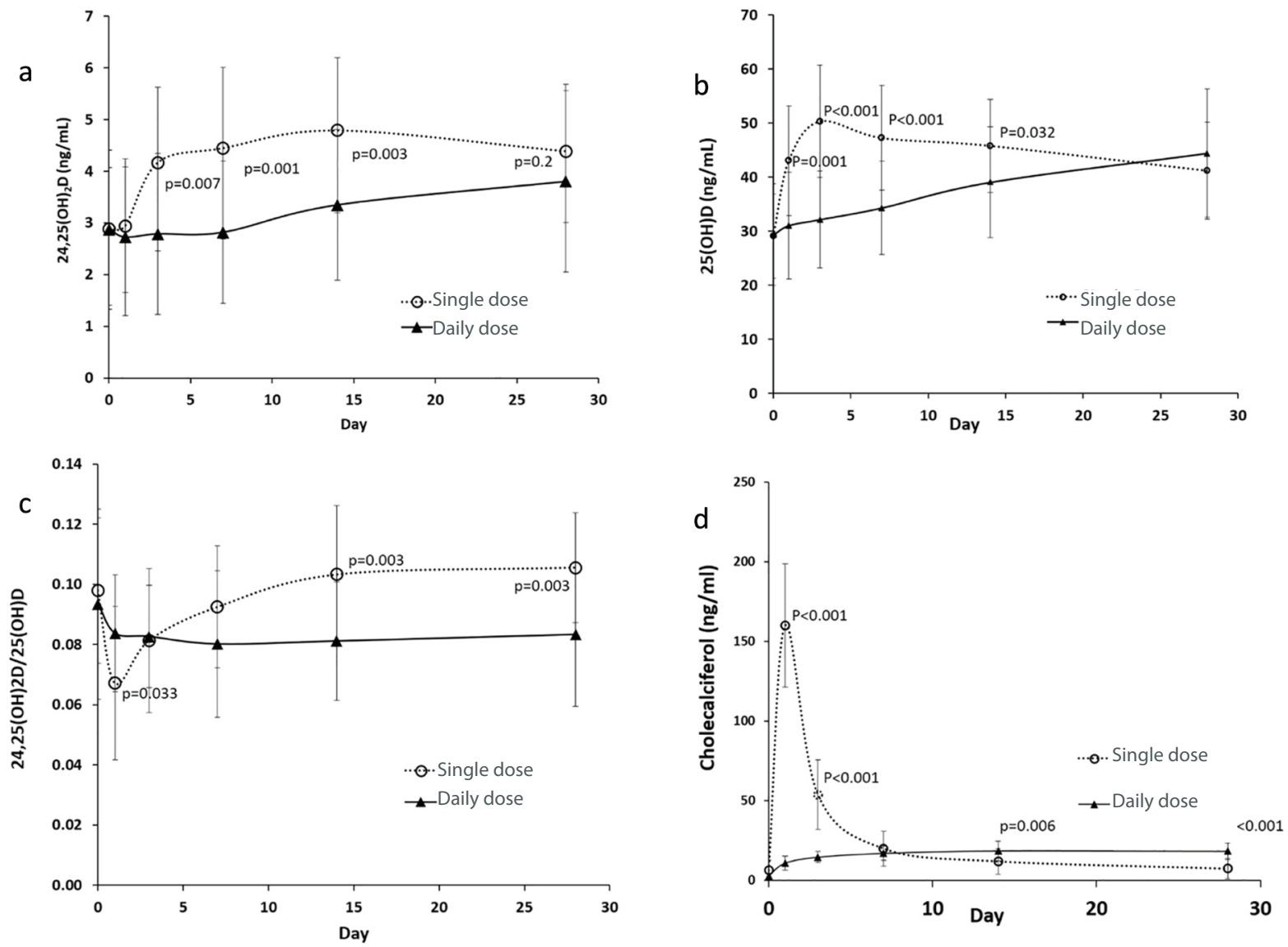

Fig 2. Alterations in (a) serum concentration of $24,25(\mathrm{OH}) 2 \mathrm{D}$, (b) serum concentration of $25(\mathrm{OH}) \mathrm{D}$, (c) ratio of $24,25(\mathrm{OH}) 2 \mathrm{D} / 25(\mathrm{OH}) \mathrm{D}$, and (d) serum concentration of cholecalciferol following a single dose of 150,000 IU vitamin D3 (open circles) and daily dose of 5000IU for 28 days (black triangles). The bars represent standard deviations. P values are for comparison of values in daily and single dosing groups at corresponding time points. It can be seen that single high-dose bolus vitamin D induces an increase in the inactivating 24 -hydroxylase activity that lasts for around 4 weeks. Reproduced with permission from Ketha et al. ${ }^{26}$ 


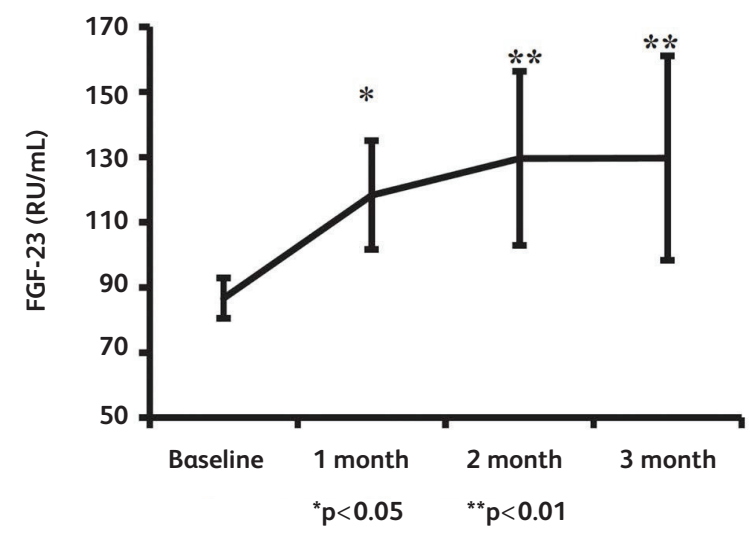

Fig 3. Changes in serum FGF23 after a single bolus of ergocalciferol (vitamin D2) 300,000 IU intramuscularly in 45 subjects with vitamin D deficiency/insufficiency. FGF23, which inhibits $1 \alpha$-hydroxylase activity, is increased by $50 \%(P<0.01) 3$ months after the bolus. Reproduced with permission from Turner et al..$^{29}$

context of hypophosphatemia is considered the cut-off for diagnosis. ${ }^{28}$ Importantly, the rise in FGF23 following vitamin D bolus is very longlasting. A single large intramuscular bolus of vitamin D2 (300,000 IU) given to 45 subjects induced a progressive $50 \%$ increase of circulating FGF23 over at least the following 3 months $(p<0.01)^{29}$ (Fig 3). FGF23 in-turn markedly suppresses $1 \alpha$-hydroxylation of $25(\mathrm{OH}) \mathrm{D}$ resulting in reduced intracellular activation of vitamin $\mathrm{D}$ to $1,25(\mathrm{OH}) 2 \mathrm{D}$ as demonstrated in monocytes. ${ }^{30}$

In studies of dialysis patients, low serum $25(\mathrm{OH}) \mathrm{D}$ combined with high serum FGF23 was shown to associate strongly with infectious and cardiac events and all-cause mortality. ${ }^{31}$

A simpler explanation might be that high-dose bolus supplementation yields blood concentrations of vitamin D that are harmful. This seems a less likely explanation for the lack of therapeutic effect of bolus supplementation, since potentially harmful blood concentrations are only approached very transiently if at all, eg in the first 24 hours after bolus administration of high-dose $25(\mathrm{OH}) \mathrm{D}^{32}$ and hypercalcaemia is rarely reported. There is also controversial population-based evidence to support a ' $U$-shaped curve' with less benefit at higher mean serum 25(OH)D concentrations. ${ }^{33}$ This is disputed though ${ }^{34}$ and seems unlikely to explain the overall negative results of so many trials of bolus supplementation. There are of course other reasons for some of the negative trial results, including vitamin $D$ being treated as a drug rather than a nutrient, often with inclusion of many participants with normal vitamin D status at baseline. ${ }^{35}$

\section{Implications for COVID-19}

There is therefore a clear biological explanation for the lack of efficacy of vitamin D supplementation when delivered as intermittent high-dose boluses. This lack of efficacy of bolus replacement is emphatically reinforced by the recent trial in rickets. ${ }^{6}$ If the literature on efficacy of vitamin D supplementation in treating or preventing various conditions including respiratory infections is reviewed with this in mind, the efficacy of regular daily maintenance vitamin D supplementation becomes much clearer and more convincing. This is borne out powerfully by the careful meta-analysis by Jolliffe, Martineau and colleagues that shows vitamin $\mathrm{D}$ supplementation is associated with significant reduction in risk for a range of acute respiratory infections but only when given as regular daily maintenance and not when given at weekly or 1-3-monthly intervals. ${ }^{17}$ Given that COVID-19 is also primarily a respiratory infection, this seems highly relevant.

We have pointed out elsewhere the links between vitamin D deficiency, latitude, seasonality, ultraviolet exposure, obesity, ethnicity and COVID-19 risk. ${ }^{1}$ There, and elsewhere, ${ }^{36}$ we have also discussed the complex impacts of illness on interpretation of circulating $25(\mathrm{OH}) \mathrm{D}$ concentrations in sick patients, the possibility of the 'healthy user' effect and the difficulties these present in drawing firm conclusions from association data. Nevertheless, the marked congruence between the risk factors for vitamin $D$ deficiency and the risk factors for severe COVID-19 make the link very plausible. This is supported by the strongly positive results of high-dose $25(\mathrm{OH}) \mathrm{D}$ (calcifediol) therapy given three times in the first week then weekly in a small Spanish trial of hospitalised patients. ${ }^{37}$ It should be noted that high-dose bolus of 25(OH)D will also likely induce potentially deleterious induction of 24-hydroxylase and FGF23, as a consequence of commensurate elevation of serum 1,25(OH)2D. ${ }^{32}$ It is plausible though that repeated dosing, particularly in the first week of therapy as used in the Spanish trial, could override this. The use of $25(\mathrm{OH}) \mathrm{D}$ is interesting and unusual. Sick patients may have reduced hepatic 25 -hydroxylation so correction of low serum $25(\mathrm{OH}) \mathrm{D}$ may be faster with $25(\mathrm{OH})$ D supplementation than with standard cholecalciferol. ${ }^{38}$ The only other randomised trial, published as a pre-print, is a negative trial from Brazil of a bolus of 200,000 IU cholecalciferol given once only to patients admitted to hospital, randomised an average of 10 days after symptom onset. ${ }^{39}$ Before the pandemic there have also been trials of vitamin $D$ in patients admitted to intensive care but unfortunately these trials (nine to date) have also used highdose bolus cholecalciferol and have all proved negative..$^{40}$ There is an ongoing international study (VITDALIZE) targeting vitamin-Ddeficient critically ill patients that also has a high frontloading bolus dose (540,000 IU cholecalciferol) but with regular 4,000 IU daily dosing afterwards for 90 days. ${ }^{41}$

NICE has noted the considerable incidence of vitamin $D$ deficiency in the UK, particularly in winter, and has recommended its supplementation 'for bone and muscle health' but has concluded that evidence is currently insufficient to recommend vitamin $D$ supplementation for preventing or treating COVID-19. We have previously pointed out the difficulty of conducting a placebocontrolled trial for a vitamin in the community. ${ }^{42}$ Waiting until the spring of 2021 at the earliest for the completion of any such trial would moreover lose the opportunity to mitigate COVID-19 risks earlier through correction of population vitamin D deficiency and the appropriate use of calcifediol in COVID-19 illness.

It seems likely to us that much of the current scepticism about the benefits of vitamin D supplementation amongst clinicians, including perhaps those on advisory committees, comes from the contradictory results of trials investigating either bolus or maintenance supplementation. We urge health professionals to note the substantial evidence for efficacy of low-dose daily maintenance vitamin $D$, rather than intermittent bolus, against various relevant clinical endpoints and to move urgently to encourage the general population to avoid vitamin D deficiency, defined as $<50 \mathrm{nmol} / \mathrm{L}$. ${ }^{22}$ They should be urged to do this to support immune function rather than just bone and muscle health. In the Northern Hemisphere vitamin D deficiency is at peak incidence 
from February to March and can be readily avoided by a daily supplement of 800-1,000 IU, preferably starting with a higher dose eg 4,000 IU/day for the first four weeks if deficiency is suspected, ${ }^{22}$ for example in people with dark skin or living in residential care, shielding indoors or working night shifts.

\section{Conflicts of interest}

Martin Hewison and David Thickett have received speaking honoraria from Thornton Ross.

\section{References}

1 Griffin G, Hewison M, Hopkin J et al. Vitamin D and COVID-19: evidence and recommendations for supplementation. Royal Soc Open Sci 2020; 7:201912.

2 National Institute for Health and Care Excellence. COVID-19 rapid guideline: vitamin D. NICE, 2020. www.nice.org.uk/guidance/ng187.

3 Autier P, Mullie P, Macacu A et al. Effect of vitamin D supplementation on non-skeletal disorders: a systematic review of meta-analyses and randomised trials. Lancet Diabetes Endocrinol 2017:5:986-1004

4 Bolland MJ, Avenell A, Grey A. Should adults take vitamin D supplements to prevent disease? BMJ 2016;355:i6201.

5 Bouillon R, Antonio L. Nutritional rickets: Historic overview and plan for worldwide eradication. J Steroid Biochem Mol Biol 2020;198:105563.

6 Crowe FL, Mughal MZ, Maroof Z et al. Vitamin D for growth and rickets in stunted children: A randomized trial. Pediatrics 2021;147:e20200815.

7 Chibuzor MT, Graham-Kalio D, Osaji JO, Meremikwu MM. Vitamin D, calcium or a combination of vitamin D and calcium for the treatment of nutritional rickets in children. Cochrane Database Syst Rev 2020;4:CD012581.

8 Thacher TD, Fischer PR, Pettifor JM. Vitamin D treatment in calcium-deficiency rickets: a randomised controlled trial. Arch Dis Child 2014;99:807-11.

9 Green M. Cod liver oil and tuberculosis. BMJ 2011;343:d7505.

10 Jarrett P, Scragg R. A short history of phototherapy, vitamin D and skin disease. Photochem Photobiol Sci 2017;16:283-90.

11 Jolliffe DA, Ganmaa D, Wejse C et al. Adjunctive vitamin D in tuberculosis treatment: meta-analysis of individual participant data. Eur Respir ] 2019;53:1802003.

12 Mily A, Rekha RS, Kamal SM et al. Significant effects of oral phenylbutyrate and vitamin D3 adjunctive therapy in pulmonary tuberculosis: A randomized controlled trial. PLoS One 2015;10: e0138340

13 Turnbull ER, Drobniewski F. Vitamin D supplementation: a comprehensive review on supplementation for tuberculosis prophylaxis. Expert Rev Respir Med 2015;9:269-75.

14 Ganmaa D, Giovannucci E, Bloom BR et al. Vitamin D, tuberculin skin test conversion, and latent tuberculosis in Mongolian school-age children: a randomized, double-blind, placebo-controlled feasibility trial. Am J Clin Nutr 2012;96:391-6.

15 Ganmaa D, Uyanga B, Zhou X et al. Vitamin D supplements for prevention of tuberculosis infection and disease. N Engl J Med 2020;383:359-68.

16 Martineau AR, Jolliffe DA, Hooper RL et al. Vitamin D supplementation to prevent acute respiratory tract infections: systematic review and meta-analysis of individual participant data. BMJ 2017;356:i6583

17 Jolliffe D, Camargo CA, Sluyter ] et al. 2020 Vitamin D supplementation to prevent acute respiratory infections: systematic review and meta-analysis of aggregate data from randomised controlled trials. medRxiv 2020.07.14.20152728.
18 Wacker M, Holick MF. Sunlight and Vitamin D: A global perspective for health. Dermatoendocrinol 2013;5:51-108.

19 Chun RF, Lauridsen AL, Suon L et al. Vitamin D-binding protein directs monocyte responses to 25-hydroxy-and 1,25-dihydroxyvitamin D. J Clin Endocrinol Metab 2010;95:3368-76.

20 Martinaityte I, Kamycheva E, Didriksen A, Jakobsen J, Jorde R. Vitamin D stored in fat tissue during a 5-year intervention affects serum 25-hydroxyvitamin D levels the following year. J Clin Endocrinol Metab 2017;102:3731-8.

21 Wu Z, Camargo CA Jr, Reid IR et al. What factors modify the effect of monthly bolus dose vitamin $D$ supplementation on 25-hydroxyvitamin D concentrations? J Steroid Biochem Mol Biol 2020;201:105687.

22 Griffin G, Hewison M, Hopkin ] et al. Preventing vitamin D deficiency during the COVID-19 pandemic: UK definitions of vitamin D sufficiency and recommended supplement dose are set too low. Clin Med 2021;21:e48-51.

23 Jones G, Prosser DE, Kaufmann M. 25-Hydroxyvitamin D-24hydroxylase (CYP24A1): its important role in the degradation of vitamin D. Arch Biochem Biophys 2012;523:9-18.

24 Kundu R, Chain BM, Coussens AK, Khoo B, Noursadeghi M. Regulation of CYP27B1 and CYP24A1 hydroxylases limits cellautonomous activation of vitamin D in dendritic cells. Eur ] Immunol 2014:44:1781-90

25 Adams JS, Chen H, Chun R et al. Substrate and enzyme trafficking as a means of regulating 1,25-dihydroxyvitamin D synthesis and action: the human innate immune response. J Bone Miner Res 2007;22:V20-4.

26 Ketha H, Thacher TD, Oberhelman SS et al. Comparison of the effect of daily versus bolus dose maternal vitamin $D_{3}$ supplementation on the 24,25 -dihydroxyvitamin $D_{3}$ to 25 -hydroxyvitamin D ratio. Bone 2018:110:321-5.

27 Zittermann A, Berthold HK, Pilz S. The effect of vitamin D on fibroblast growth factor 23: a systematic review and meta-analysis of randomized controlled trials. Eur ] Clin Nutr 2020. doi: 10.1038/ s41430-020-00725-0.

28 Florenzano P, Hartley IR, Jimenez M et al. Tumor-induced osteomalacia. Calcif Tissue Int 2021;108:128-42.

29 Turner C, Dalton N, Inaoui R et al. Effect of a 300 000-IU loading dose of ergocalciferol (Vitamin D2) on circulating 1,25(OH)2vitamin D and fibroblast growth factor-23 (FGF-23) in vitamin D insufficiency. J Clin Endocrinol Metab 2013;98:550-6.

30 Bacchetta J, Sea JL, Chun RF et al. Fibroblast growth factor 23 inhibits extrarenal synthesis of 1,25-dihydroxyvitamin D in human monocytes. J Bone Miner Res 2013;28:46-55.

31 Chonchol M, Greene T, Zhang Y, Hoofnagle AN, Cheung AK. Low vitamin D and high fibroblast growth factor 23 serum levels associate with infectious and cardiac deaths in the HEMO study. J Am Soc Nephrol 2016;27:227-37.

32 Petkovich M, Melnick J, White J et al. Modified-release oral calcifediol corrects vitamin D insufficiency with minimal CYP24A1 upregulation. J Steroid Biochem Mol Biol 2015;148:283-9.

33 Amrein K, Quraishi SA, Litonjua AA et al. Evidence for a U-shaped relationship between prehospital vitamin D status and mortality: a cohort study. J Clin Endocrinol Metab 2014;99:1461-9.

34 Fan X, Wang J, Song M et al. Vitamin D status and risk of all-cause and cause-specific mortality in a large cohort: results from the UK Biobank. J. Clin. Endocrinol. Metab 2020;105: e3606-19.

35 Boucher BJ. Why do so many trials of vitamin D supplementation fail? Endocr Connect 2020;9:R195-6.

36 Rhodes JM, Subramanian S, Laird E, Griffin G, Kenny RA. Perspective: Vitamin D deficiency and COVID-19 severity plausibly linked by latitude, ethnicity, impacts on cytokines, ACE2 and thrombosis. J Intern Med 2021;289:97-115.

37 Castillo ME, Entrenas Costa LM, Vaquero Barrios JM et al. Effect of calcifediol treatment and best available therapy versus best 
available therapy on intensive care unit admission and mortality among patients hospitalized for COVID-19: A pilot randomized clinical study. J Steroid Biochem Mol Biol 2020;203:105751.

38 Quesada-Gomez JM, Bouillon R. Is calcifediol better than cholecalciferol for vitamin D supplementation? Osteoporos Int 2018;29:1697-1711.

39 Murai IH, Fernandes AL, Sales LP et al. Effect of vitamin $D_{3}$ supplementation vs placebo on hospital length of stay in patients with severe COVID-19: A multicenter, double-blind, randomized controlled trial. medRxiv 2020.11.16.20232397.

40 Peng L, Li L, Wang P, Chong W et al. Association between Vitamin D supplementation and mortality in critically ill patients: A systematic review and meta-analysis of randomized clinical trials. PLoS One 2020;15:e0243768.

41 Amrein K, Parekh D, Westphal S et al. Effect of high-dose vitamin D3 on 28-day mortality in adult critically ill patients with severe vitamin $D$ deficiency: a study protocol of a multicentre, placebo-controlled double-blind phase III RCT (the VITDALIZE study). BMJ Open 2019;9:e031083.

42 Griffin G, Hewison M, Hopkin ] et al. Rapid Response: re: vitamin D and COVID-19 - this is no time for procrastination. BMJ 22 December 2020; www.bmj.com/content/371/bmj.m4912/rapidresponses.

Address for correspondence: Prof Jonathan Rhodes,

Department of Cellular and Molecular Physiology, Institute of Translational Medicine, Henry Wellcome Laboratory, Nuffield Building, Crown Street, Liverpool L69 3GE, UK.

Email: rhodesjm@liverpool.ac.uk 